\title{
LabTEAR: a colaboração como principio para o desenvolvimento de novas metodologias apoiadas no uso de TICS
}

\author{
Márcia Cristina Moraes ${ }^{1,3}$, Leticia Lopes Leite ${ }^{2,3}$, Rosana Maria Gessinger ${ }^{2,3}$, Sônia \\ Maria de Souza Bonelli ${ }^{2,4}$, Ana Lúcia Souza de Freitas ${ }^{2,3}$, Afonso Strehl ${ }^{2,3}$, Valderez \\ Marina do Rosário Lima ${ }^{2,3}$ \\ ${ }^{1}$ Faculdade de Informática, PUCRS, Brasil \\ ${ }^{2}$ Faculdade de Educação, PUCRS, Brasil \\ ${ }^{3}$ Pró-Reitoria de Graduação, PUCRS, Brasil \\ ${ }^{4}$ Coordenadoria de Educação a Distância, PUCRS, Brasil \\ \{marcia.moraes, leticia.leite, rosana.gessinger, sbonelli, ana.freitas, afontrel, \\ valderez.lima\}@pucrs.br
}

Resumo. O texto tematiza o diálogo entre as Tecnologias de Informação e Comunicação (TICs) e a Educação Superior, enfatizando a necessidade de desenvolvimento de novas abordagens metodológicas para favorecer a aprendizagem colaborativa. Apresenta o projeto de criação do LabTEAR e detalha a experiência de seu primeiro subprojeto - o LABS MÓVEIS. Ao final, refere os fundamentos e perspectivas para a continuidade desses projetos.

Palavras-chaves: Aprendizagem em rede, Mobilidade, Tecnologias de Informação e Comunicação como Apoio ao Ensino

\section{LabTEAR: collaboration as the principle for developing new methods supported by the use of ICT}

Abstract. The paper aims to discuss the dialogue between Information and Communication Technologies (ICTs) and Higher Education, emphasizing the need to develop new methodological approaches to encourage collaborative learning. It presents the design and creation of LabTEAR and details the experience of his first subproject the LabsMóveis. Finally, describes the fundamentals and prospects to projects' development.

Keywords: Networked learning, Mobility, Information and Communication Technologies as Education Support

\section{Introdução}

$\mathrm{Na}$ atual sociedade do conhecimento (UNESCO, 2009), interconectada em redes de crescente complexidade, viabilizadas por recursos tecnológicos sempre mais aperfeiçoados, espera-se que a universidade exerça um papel de protagonismo. De forma cada vez mais intensa, no cumprimento de seu papel na educação superior, no âmbito do ensino, da pesquisa e da extensão, a universidade vale-se de diferentes 
Tecnologias de Informação e Comunicação, aplicando-as à produção e difusão do conhecimento.

Ito et. al (2009) apresentam alguns conceitos que caracterizam a maneira como os jovens vivem e aprendem com as novas mídias. Dentre eles, podemos destacar a ecologia das novas mídias e as redes públicas. Por novas mídias os autores entendem a maneira como os meios mais tradicionais, como livros, televisão e rádio, estão interconectados com os meios digitais, especialmente as mídias interativas e redes online de comunicação social. A palavra ecologia é utilizada para enfatizar que as atividades realizadas pelos jovens estão dinamicamente conectadas com as tecnologias. Em relação ao termo redes públicas, os autores descrevem a participação ativa das redes sociais na produção e divulgação da cultura e do conhecimento.

Tendo em mente esses conceitos, entende-se que a ampliação do uso de tecnologias no processo de ensino e de aprendizagem aproxima a cultura acadêmica à linguagem dos jovens, mantendo o foco no essencial que, conforme Lévy (2000, p. 158), é representado por "um novo estilo de pedagogia, que favorece ao mesmo tempo as aprendizagens personalizadas e a aprendizagem coletiva em rede. Nesse contexto, o professor é incentivado a tornar-se um animador da inteligência coletiva de seus grupos de alunos em vez de um fornecedor direto de conhecimentos”.

Dentre o conjunto das ações em desenvolvimento na Universidade para estimular o uso de TICs na aula universitária, destaca-se o Projeto Laboratório de Tecnologias para Aprendizagem em Rede (LabTEAR), que tem como objetivo geral produzir metodologias que incorporem o uso de TICs para favorecer a aprendizagem colaborativa, conectando a aula universitária da Universidade em redes de conhecimento.

O projeto LabTEAR se alinha às conclusões da UNESCO, expressas no comunicado final da Conferência Mundial sobre Educação Superior, realizada em Paris no ano de 2009. O referido documento indica a inclusão, na agenda da educação global da sociedade do conhecimento, de "polos e redes de pesquisa de excelência, inovações no ensino/aprendizagem e novas abordagens para a extensão comunitária” (UNESCO, 2009).

Deste modo, esse artigo tem como objetivo apresentar o projeto LabTEAR, relatar uma experiência de uso de uma metodologia que utiliza TICs na aula de graduação e apresentar perspectivas e considerações para a continuidade do projeto.

\section{A criação do Projeto LabTEAR}

O Projeto LabTEAR apresenta-se como uma proposta institucional para responder aos desafios inerentes ao diálogo entre as Tecnologias da Informação e Comunicação e a docência no ensino superior. De forma mais específica, o Projeto tem como objetivos conhecer as possibilidades do uso de recursos de tecnologia para apoio ao ensino; criar laboratórios de TICs para qualificar a gestão da aula universitária na Universidade; elaborar propostas pedagógicas para a utilização de TICs, em consonância com o Projeto Pedagógico Institucional e com os objetivos estratégicos da Universidade; investigar as repercussões das propostas pedagógicas elaboradas tendo em vista sua ampliação. A fim de atingir seus objetivos, o projeto LabTEAR se organiza nas seguintes fases: implantação, implementação e avaliação. 


\subsection{Implantação do LabTEAR}

A fase de implantação se refere à etapa na qual são realizadas definições acerca das necessidades envolvidas no projeto. Por ser um laboratório que visa à aprendizagem colaborativa por meio da construção de redes de conhecimento, uma das características fundamentais para o desenvolvimento do trabalho é a definição de uma estrutura física que possibilite a interação, a mobilidade e a construção coletiva.

O laboratório foi inaugurado em dezembro de 2012 e conta com oito possibilidades de layouts flexíveis que permitem a realização de diferentes práticas pedagógicas, tais como seminários, palestras, trabalhos em pequenos e grandes grupos. Tendo a mobilidade como um dos princípios orientadores, o laboratório foi equipado com tecnologias móveis, como tablets e notebooks. O laboratório também possui uma lousa eletrônica e uma smart TV, através dos quais podem ser realizadas videoconferências.

Aliado ao princípio da mobilidade, a constituição de um grupo de estudos que contempla as áreas das ciências exatas, humanas e sociais, subsidia a pesquisa sobre a aplicação deste projeto em diferentes contextos de educação.

\subsection{Implementação do LabTEAR}

A fase de implementação se constitui da efetiva realização do projeto, por meio do desenvolvimento de subprojetos a serem definidos pelo grupo de estudos, visando a construção de propostas metodológicas voltadas ao ensino de graduação, em diferentes áreas, de modo a favorecer a aprendizagem colaborativa a partir do uso das TICs.

Atualmente, encontra-se em execução o subprojeto denominado LabsMóveis, detalhado na seção três.

\subsection{Avaliação do LabTEAR}

A fase de avaliação se constitui: do acompanhamento do processo de implementação dos subprojetos; da avaliação dos subprojetos e de suas contribuições para a aprendizagem, considerando as propostas pedagógicas elaboradas e da tomada de decisões sobre as possibilidades de ampliação das propostas pedagógicas elaboradas.

\section{Subprojeto LabsMóveis}

O subprojeto LabsMóveis tem como objetivo geral elaborar, aplicar e avaliar estratégias de ensino adequadas ao uso de tecnologias em aulas de graduação. Estão envolvidas no projeto as seguintes Faculdades: Educação (FACED); Direito (FADIR); Enfermagem, Nutrição e Fisioterapia (FAENFI); Física (FAFIS); Comunicação Social (FAMECOS) e Engenharia (FENG). Com foco nos objetivos do subprojeto, as Faculdades indicaram os docentes que iriam participar do grupo de estudos. Estes, por sua vez, selecionaram as disciplinas para as quais seriam elaboradas, aplicadas e avaliadas as estratégias de ensino. Além dos professores das Faculdades mencionadas, participam do projeto consultores da área de tecnologias e também a equipe da Coordenadoria de Ensino e Desenvolvimento Acadêmico (CEDA) da Pró-Reitoria Acadêmica da Universidade.

As próximas seções apresentam, de maneira breve, o trabalho desenvolvido ao longo do ano de 2012 e início de 2013 e a descrição de uma metodologia produzida pela professora da FACED que integra o grupo de estudos. 


\subsection{Desenvolvimento do Subprojeto LabsMóveis}

Inicialmente, foram realizadas reuniões semanais com a equipe de professores, a equipe da CEDA e os consultores para a definição dos recursos de hardware e software mais apropriados a cada uma das Faculdades participantes. O objetivo das reuniões com os consultores era apresentar as propostas pedagógicas que seriam desenvolvidas pelas Faculdades e realizar discussões acerca dos recursos mais adequados para cada situação. Neste sentido, algumas Faculdades optaram por utilizar notebooks, enquanto outras, por utilizar IPADs.

Após a definição dos recursos a serem utilizados, foi elaborada uma agenda de encontros quinzenais envolvendo o grupo de professores. Estas reuniões tiveram como enfoque o estudo de conceitos, a elaboração preliminar de princípios da mediação pedagógica na aula universitária, o desenvolvimento de práticas pedagógicas pelas Faculdades integrantes do LabsMóveis e a apropriação das ferramentas indicadas para a sua implementação.

A referida apropriação foi realizada a partir de dois momentos de capacitação. O primeiro foi constituído da apresentação de diferentes aplicativos para IPADs, que podem ser utilizados, tanto por professores quanto por alunos, para construção de materiais e realização de dinâmicas na sala de aula. O segundo momento foi constituído de um relato de estudo sobre as principais redes sociais atualmente sendo utilizadas pelos alunos. Durante o relato, houve diversas discussões sobre as dificuldades e possibilidades dos usos dessas ferramentas.

Após a realização das capacitações, os professores foram convidados a elaborar uma proposta de dinâmica do uso dos recursos móveis em aula. Estas atividades foram vivenciadas pelos demais professores envolvidos no subprojeto. O desenvolvimento da primeira atividade prática ficou sob a responsabilidade dos professores da FAENFI. Durante a sua realização, o grupo deveria verificar quais princípios do projeto estavam sendo contemplados na atividade e, ao final, promoveu-se uma discussão acerca do trabalho realizado.

A segunda atividade foi de responsabilidade dos professores da FACED e FENG que realizaram uma atividade envolvendo o uso do YouTube, Facebook e Moodle. Seguindo a mesma dinâmica de realização da atividade proposta pela FAENFI, o grupo observou os princípios que estavam presentes na realização da atividade e, ao final da mesma, promoveu uma discussão acerca da experiência.

A partir da vivência das práticas pedagógicas, constatou-se a necessidade de sistematizá-las, deixando registradas as estratégias de ensino elaboradas pelo grupo, com vistas à possibilidade de divulgar posteriormente aos demais docentes da Universidade.

O último encontro do primeiro semestre ficou sob a responsabilidade dos professores da FADIR, que elaboraram uma atividade pedagógica envolvendo o uso do GoogleDocs e do Moodle.

Salienta-se que ao final de cada encontro, os professores apresentavam um relato sobre o uso dos recursos com alunos de graduação das disciplinas participantes do subprojeto.

Para dar suporte às atividades de gestão, foram criadas duas áreas para o compartilhamento de informações acerca das atividades do Projeto. A primeira área, 
nomeada LabsMóveis, foi criada no Ambiente Moodle da Instituição. Neste espaço estão disponíveis as ferramentas de comunicação, wikis, diários e materiais produzidos pelo grupo de estudos, tais como detalhamento do projeto, princípios orientadores, cronograma de encontros e termo de consentimento livre e esclarecido a ser aplicado com os alunos das disciplinas envolvidas.

A segunda área, nomeada GRUPO LabsMóveis, foi criada como um grupo fechado do Facebook, a partir da demanda dos professores. Inicialmente, este espaço deveria ser utilizado em uma das propostas pedagógicas, entretanto tornou-se o espaço prioritário para as postagens do grupo de professores.

A realização das atividades propostas pelas Faculdades possibilitou aos professores participantes colocar-se no lugar de aprendizes e vislumbrar, na prática, as contribuições das TICs para a efetivação dos princípios da mediação pedagógica na aula universitária. O trabalho desenvolvido resultou na proposição e realização de uma oficina intitulada "Tablets, notebooks e outros equipamentos móveis na dinâmica da aula universitária”, durante o Seminário de Capacitação Docente da Universidade, no primeiro semestre de 2012. Desse modo, foi possível vislumbrar, desde a fase inicial, a fecundidade do subprojeto LabsMóveis e suas contribuições para o Projeto LabTEAR.

As reuniões com o grupo de estudos continuaram ao longo do segundo semestre de 2012, tendo como enfoque a produção, aplicação e sistematização de práticas pedagógicas. Para isso, inicialmente foram escolhidos dois procedimentos didáticos, problematização e pesquisa em sala de aula, e os professores envolvidos no projeto planejaram atividades que contemplassem o uso dos dispositivos móveis em tais procedimentos. Nas reuniões seguintes, os docentes relataram a realização das atividades pelos alunos, e o grupo analisou criticamente as experiências relatadas, com vistas à sistematização do conhecimento produzido ao longo do semestre. Como parte dos resultados deste segundo semestre de trabalhos, o grupo apresentou no Seminário de Capacitação Docente da Universidade, em dezembro, um relato de experiência seguido de um grupo de discussão intitulado "LabTEAR: tecendo o diálogo entre as TICs e a Educação Superior”.

No ano de 2013 as reuniões do grupo continuam a ocorrer quinzenalmente e têm como foco a análise e avaliação dos procedimentos didáticos aplicados nas diferentes turmas que fazem parte do projeto.

3.2. A WebQuest como recurso para a aula de graduação na formação de professores da Educação Infantil e dos Anos Iniciais do Ensino Fundamental

A WebQuest é uma atividade dirigida à aprendizagem, com apoio na pesquisa, na qual algumas ou todas as informações estão disponíveis na Internet para os alunos utilizarem. (DODGE (1995) APUD BARBOSA E ABAR 2008). O autor, ao propor este conceito, apresenta uma revolução no âmbito educacional, uma vez que nos suscita questionar o uso da WEB 2.0 na Educação. Tais questionamentos podem ser representados por diferentes perguntas, tais como: será que com a utilização da internet meu aluno vai aprender melhor? A aprendizagem será mais significativa? Como avaliar o resultado a partir desta abordagem?

Podemos dizer que ainda não temos todas as respostas, mas também podemos afirmar que a utilização dos recursos da WEB 2.0 torna a aula mais dinâmica e interativa. A WebQuest, em especial, permite o movimento da Pequisa em Sala de Aula, V. $11 \mathrm{~N}^{\circ} 1$, julho, 2013 
onde temos o primeiro desafio que é o problema, seguido pela argumentação, ou pesquisa propriamente dita, e a comunicação, ou seja a apresentação das soluções para a questão levantada. (MORAES e LIMA, 2002)

Nesse sentido, e com o compromisso de melhorar a aula da graduação, no segundo semestre de 2012 trabalhamos com esta proposta na disciplina de Princípios e Propostas Metodológicas de Ciências. O objetivo da tarefa era que os alunos realizassem uma pesquisa colaborativa com acesso à informação atualizada e utilizassem as WebQuests produzidas com seus alunos dos Anos Iniciais, oportunizando às crianças mais uma possibilidade de aprender Ciências de forma prazerosa e aos alunos de desenvolver o espírito crítico através da investigação científica. Para a realização desta atividade, utilizamos os IPads do Projeto LabsMóveis.

Tendo em vista apresentar aos alunos um exemplo de WebQuest, desenvolvemos o enunciado do trabalho no formato de uma WebQuest. Neste sentido, a estrutura da WebQuest que representa o enunciado do trabalho possuiu organização apresentada abaixo.

\section{INTRODUÇÃO}

- A WebQuest permite uma atividade investigativa em aula; acesso à informação atualizada e também a aprendizagem colaborativa.

- Compartilha saberes pedagógicos como ferramenta aberta de cooperação e intercâmbio docente.

- Favorece um trabalho de autoria dos professores e dos alunos.

- Desenvolve habilidades como aprender a aprender e aprender a fazer.

\section{TAREFA}

- A partir dos textos trabalhados em aula, em especial a problematização e a pesquisa em sala de aula organize uma WebQuest com o conteúdo sorteado em aula.

- Utilize a internet como fonte de pesquisa.

\section{PROCESSO}

- Organizar grupos de trabalho conforme o conteúdo sorteado.

- Estudar o tema proposto e elaborar a WebQuest.

- Ajustar a WebQuest à série estabelecida, neste caso o quinto ano do Ensino Fundamental.

- $\quad$ Apresentar a WebQuest na forma de PPT ou outra organização.

\section{RECURSO}

- Para realizar a atividade faça uso dos seguintes links.

- Exemplos de WebQuest

http://www.emack.com.br/sao/webquest/webquestsp.php

http://webquesst.blogspot.com.br/

http://www.cienciamao.usp.br/index.php

http://www.youtube.com/watch?v=WUnig_lAjF8

AVALIAÇÃO

- Serão considerados para a avaliação do trabalho os seguintes critérios:

- Envolvimento com o grupo estando presente em todas as aulas.

- Consistência da atividade elaborada.

- Adequação com a turma em que será aplicado.

- Utilização adequada dos conceitos estudados. 


\section{CONCLUSÃO}

- O uso da WebQuest tem por objetivo a pesquisa colaborativa e acesso a informação atualizada, entre outros.

- Dessa forma, utilizar esta proposta com os alunos dos Anos Iniciais é oportunizar às crianças mais uma possibilidade de ensinar Ciências de forma prazerosa e também fazer com que eles desenvolvam o espírito crítico através da investigação científica.

Os alunos tiveram quatro encontros de $4 \mathrm{~h}$ para realizar a atividade proposta e, ao final, apresentaram para o grupo todo. Podemos afirmar que o envolvimento do grupo foi significativo, pois estavam presentes em todas as aulas, pesquisaram além do material proposto, trouxeram exemplos e também avaliaram sobre a importância de o professor saber o conteúdo para poder organizar uma atividade como a WebQuest.

Um dos resultados pode ser visto no endereço: http://www.webquestbrasil.org/criador2/webquest/soporte_tabbed_w.php?id_actividad= 18497\&id_pagina=1.

Através de depoimentos dos alunos como "a aula ficou mais significativa”; "no início foi difícil, mas o resultado foi compensador”; “o desafio nos fez ir além”, entre outros, podemos perceber que o resultado foi muito positivo.

\section{Projeto LabTEAR: fundamentos e perspectivas}

O diálogo entre as Tecnologias da Informação e Comunicação (TICs) e a docência no ensino superior anuncia-se como um desafio da contemporaneidade, diante de um cenário em que os estudantes, cada vez mais, fazem uso de ferramentas tecnológicas para o estabelecimento de relações sociais, em diferentes instâncias.

Nesse âmbito, a intensidade com que as redes sociais e outras ferramentas tecnológicas têm se incorporado ao cotidiano dos acadêmicos repercute nas relações de ensinar e de aprender. Ao manifestarem um domínio tecnológico que o professor ainda não tem, eles problematizam a tradicional assimetria das relações entre ambos, caracterizada pela desigualdade na apropriação do conhecimento específico. O domínio tecnológico altera tal assimetria em favor do estudante. Isso se torna mais evidente quando a ação do professor restringe-se à apresentação da informação, já que as possibilidades de acesso, por meio das TICs, são incomparáveis a qualquer capacidade humana de exposição de um conteúdo.

Desse modo, a tecnologia dá visibilidade aos limites da atuação docente reduzida à instrução, com base na exposição da informação, reinaugurando a discussão sobre as finalidades da função docente, de modo que, na atualidade, não é possível pensar um projeto pedagógico sem levar em conta, simultaneamente, as intencionalidades pedagógicas e a sua operacionalização tecnológica (TRINDADE; COSME, 2010).

Nesse sentido, merece destaque a reflexão acerca do reconhecimento de que a expansão do uso de recursos tecnológicos em aula emerge como uma necessidade que, ao se concretizar, pode contribuir para a qualificação da ação educativa. No entanto, não basta ter uma sala de aula equipada com muitos e atualizados recursos tecnológicos; é 
necessário que haja uma proposta pedagógica consistente, em consonância com o Projeto Pedagógico Institucional, que dê sustentação ao trabalho realizado com os estudantes. É neste sentido que o questionamento sobre as contribuições das TICs para qualificar a gestão da aula universitária se apresenta como objeto de investigação do Projeto LabTEAR.

O projeto LabTEAR, em consonância com o Projeto Pedagógico Institucional da Universidade (PPI), apoia-se nos pressupostos da pedagogia relacional (BECKER, 2001) e do educar pela pesquisa (DEMO, 2007) para argumentar a compreensão de que

\begin{abstract}
o ensino consiste na organização de situações capazes de contribuir para a construção e produção do conhecimento pelo aluno, distanciando-se da ideia de transferência de informação. A aprendizagem, mais do que acúmulo de informações, é compreendida como construção de significados que permitam a interpretação da realidade e sua transformação. A avaliação é um componente de diagnóstico e de reorientação do ensino e da aprendizagem, numa perspectiva de compreensão da prática docente e da trajetória acadêmica do aluno (PPI, 2012, p.12).
\end{abstract}

A continuidade dos estudos tem como perspectiva a elaboração de princípios de gestão da aula de graduação à luz das Tecnologias da Informação e Comunicação para orientar a prática educativa dos Projetos Pedagógicos dos Cursos de Graduação. Compreende-se que as experiências em andamento com as Faculdades colaboradoras do subprojeto LabsMóveis contribuirão para reiterar as concepções pedagógicas orientadoras da educação superior na instituição, expressas no PPI, bem como para ampliar as possibilidades de sua efetivação na dinâmica da aula universitária, mediante a observação e a análise de desempenho de aprendizagem e inovação.

\title{
5. Considerações para a continuidade da ação-reflexão-investigação
}

A experiência do trabalho com a equipe responsável pelo subprojeto LabsMóveis, realizada no primeiro e segundo semestres de 2012, foi bastante significativa porque permitiu perceber - ainda que em menor escala - muitos aspectos que estarão presentes na interação com os alunos, bem como nas interações entre eles. Entre esses aspectos, podemos citar a dispersão inicial diante da novidade do equipamento e da curiosidade em descobrir suas funcionalidades. Apesar de este momento inicial ser mais propício a descobertas e à curiosidade, acredita-se que a presença dos recursos tecnológicos, inevitavelmente, problematizará a dinâmica das relações de ensinar e de aprender, atribuindo novas características à aula de graduação.

Preliminarmente, apresentamos algumas considerações acerca da problematização das interações proporcionadas pelas experiências com o grupo do LabsMóveis, objetivando apoiar a continuidade dos estudos e a elaboração de propostas metodológicas.

1. Sobre as interações livres: as interações livres, mobilizadas inicial e espontaneamente, permitem perceber o potencial da interação aluno-aluno e suscitaram a reflexão sobre a necessidade/possibilidade de prever, na organização do plano de trabalho da disciplina, um tempo para o conhecimento exploratório das potencialidades do recurso tecnológico a ser utilizado. Nesse sentido, para além da curiosidade inicial, é importante considerar como esta poderá ser uma característica da aula, em outros momentos também, alterando a 
dinâmica presencial, que muitas vezes pressupõe a centralidade no educador na exposição do conteúdo, e enfatizando a mediação pedagógica como sua função.

2. Sobre a simultaneidade das interações: a possibilidade de acessar a informação de formas alternativas à fala do professor abre espaço para a realização simultânea de outras ações. Assim, o tempo de aula é também um tempo de outras descobertas e aprendizagens, para além das previstas pelo educador. Tal flexibilidade permite que os alunos trabalhem em diferentes ritmos, mas torna mais evidente a necessidade de o professor assumir a diretividade do processo, retomando as proposições feitas e proporcionando sínteses coletivas.

3. Sobre a complementaridade das relações formais e informais: as relações interpessoais do professor com os alunos contribuem para a criação de condições para a aprendizagem. Nesse sentido, as redes sociais cumprem função importante para além da sala de aula. A utilização, pelo professor, dos recursos familiares ao aluno em sua experiência cotidiana, favorece a aproximação interpessoal e contribui para a aprendizagem. O desafio proposto para pensarmos/realizarmos diz respeito às possibilidades de fazermos uso pedagógico das redes sociais e, assim, contribuir para significar as relações de aprendizagem.

4. Sobre o uso do Moodle: a importância de utilização do ambiente, por parte do professor é outro aspecto destacado, considerando que é quase inevitável admitir a presença e a necessidade das TICs nas relações de ensinar e de aprender. Então, a expectativa é a de que o Moodle se generalize como ferramenta de gestão da aula universitária na Universidade. O desafio que emerge dessa discussão é não apenas ampliar as possibilidades de uso do Moodle pelos professores, mas também o de qualificá-lo, na experiência das diferentes áreas de formação, em seu potencial de interatividade nas relações entre professor, aluno e conhecimento.

5. Sobre a questão do controle: a problematização do controle do professor é um dos impactos das TICs para as relações de ensino e de aprendizagem. O conhecimento que o aluno traz em relação ao uso das TICs põe em questão a tradição das relações entre professor e alunos, fundada na hierarquia do conhecimento de um sobre os outros. Esta "perda do controle” do professor diante das TICs é uma percepção inicial que se desfaz, por exemplo, pelo conhecimento das ferramentas do Moodle, diante da constatação de que o relatório de atividades pode proporcionar um controle muito maior do que qualquer alternativa presencial. O desafio que emerge dessa discussão é o de transformar o controle tecnicamente proporcionado pela ferramenta como forma de acompanhamento e mediação pedagógica para a promoção da aprendizagem.

6. Sobre a questão da heterogeneidade: as diferentes realidades de acesso aos recursos tecnológicos contribuem para acentuar a heterogeneidade que caracteriza as turmas de graduação. Está cada vez mais difícil se sustentar a aula como uma proposição única, organizada a partir de um pressuposto "padrão médio" de aproveitamento; a necessidade de contemplar as diferenças se apresenta como uma necessidade cada vez mais evidente. O desafio que emerge de tal discussão é o de fazer uso das TICs para assumir a diversidade como um componente planejado na ação do educador. Esta perspectiva corrobora com a 
afirmação de Davidson e Goldberg (2010) de que não existe uma aprendizagem padrão aplicável a todos os alunos.

Tais considerações expressam as exigências vislumbradas para qualificar a gestão da aula universitária. A continuidade da ação-reflexão-investigação, tendo em vista a aplicação, análise e avaliação das propostas metodológicas desenvolvidas pelo grupo do LabsMóveis, é o desafio que se apresenta neste momento.

\section{Referências}

ABAR, Celina A. A.P.; BARBOSA, Lisbete M. Webquest: um desafio para o professor. São Paulo: Avercamp. 2008

BECKER, F. Educação e construção do conhecimento. Porto Alegre: Artmed, 2001. DAVIDSON, C. N.; GOLDBERG, D. T. The Future of Thinking: Learning Institutions in a Digital Age. MIT Press, 2010.

DEMO, P. Educar pela pesquisa. 8. ed. São Paulo: Autores Associados, 2007.

ITO, M.; HORST, H.; BITTANTI, M.; BOYD, D.; HERR-STEPHENSON, B.; LANGE, P. G.; PASCOE, C. J.; ROBINSON, L. Living and Learning with New Media. Massachusetts: MIT Press, 2009.

LÉVY, Pierre. Cibercultura. 2. ed. São Paulo: Editora 34, 2000.

MORAES, R.; LIMA, V. M. R. Pesquisa em sala de aula: tendências para a educação em novos tempos. Porto Alegre: EDIPUCRS, 2002.

TRINDADE, Rui; COSME, Adriana. Todas as aprendizagens são pessoais, mas ninguém aprende sozinho: gerir as salas de aula como comunidades de aprendizagem. Curitiba: Editora Mello, 2010.

UNESCO. 2009. World Conference on Higher Education: The New Dynamics of Higher Education and Research for Societal Change and Development. Communiqué. Paris, $\quad 5 \quad-\quad-8 \quad$ July 2009 . $<$ http://www.unesco.org/fileadmin/MULTIMEDIA/HQ/ED/ED/pdf/WCHE_2009/FINA L\%20COMMUNIQUE\%20WCHE\%202009.pdf>. Acesso em: 17 jul. 2012. 